\title{
Case Report \\ Cardiac Calcified Amorphous Tumor of the Mitral Valve Presenting as Transient Ischemic Attack
}

\author{
Mohammad Abbasi Teshnizi, ${ }^{1}$ Atefeh Ghorbanzadeh, ${ }^{2}$ Nahid Zirak, \\ Babak Manafi, ${ }^{4}$ and Aliasghar Moeinipour ${ }^{1}$ \\ ${ }^{1}$ Department of Cardiac Surgery, Imam Reza Hospital, Atherosclerosis Prevention Research Center, \\ Faculty of Medical Science, Mashhad University of Medical Sciences, Mashhad, Iran \\ ${ }^{2}$ Student Research Committee, Mashhad University of Medical Sciences, Mashhad, Iran \\ ${ }^{3}$ Faculty of Medicine, Mashhad University of Medical Sciences, Mashhad, Iran \\ ${ }^{4}$ Faculty of Medicine, University of Medical Sciences, Hamedan, Iran \\ Correspondence should be addressed to Aliasghar Moeinipour; moinipooral@mums.ac.ir
}

Received 12 August 2016; Revised 12 December 2016; Accepted 4 January 2017; Published 17 January 2017

Academic Editor: Assad Movahed

Copyright ( 2017 Mohammad Abbasi Teshnizi et al. This is an open access article distributed under the Creative Commons Attribution License, which permits unrestricted use, distribution, and reproduction in any medium, provided the original work is properly cited.

Cardiac calcified amorphous tumors (CATs) are an extremely rare nonneoplastic intracardiac masses. They have been reported in the literature in only a few cases. Thus, the incidence, pathogenesis, and best approach to the treatment are not certain. We report a case of CATs on the atrial surface of the anterior mitral valve leaflet in a 37-year-old female who was diagnosed by histopathological examination after surgical removal.

\section{Introduction}

Calcified amorphous tumors (CATs) of the heart are an exceedingly rare nonneoplastic intracardiac mass that was originally described in 1997 by Reynolds et al. [1]. The clinical features of cardiac CAT are usually like the other cardiac masses which include the symptoms related to obstruction or embolization such as dyspnea and syncope. Thus, it may be misdiagnosed with other cardiac tumors [2,3]. Accurate diagnosis of a cardiac mass is often made on surgical excision and histopathological examination [1-3]. Currently, only a few cases of cardiac CATs have been reported in the literature. The incidence, pathogenesis, and best approach to the treatment are not certain. Herein, we describe a case of cardiac CATs originating from the mitral valve in a 37-yearold woman revealed by the transient ischemic attack (TIA).

\section{Case Presentation}

A 37-year-old woman sought neurological assistance after an episode of TIA manifesting predominantly as left hemiparesis of 5-minute duration. At that time, she had no abnormalities on examination of the cranial nerves, and brain computed tomography (CT) scan findings were normal. She was referred to a cardiologist to be evaluated for TIA. The physical examination and routine blood laboratory investigations results were normal. Her past medical history showed hypertension (HTN) and hyperlipidemia (HLP) which both were under control. During further clinical assessments, transesophageal echocardiography (TEE) was performed, which revealed an echogenic, round, and mobile mass measuring $5 \times 5 \mathrm{~mm}$ in diameter attached by a short pedicle on the atrial surface of the anterior mitral valve leaflet (Figure 1). There was mild to moderate regurgitation of the mitral valve. The other valves and cardiac structures were normal. Left ventricular ejection fraction (EF) was $60 \%$. The patient was given anticoagulant therapy and referred for surgery with clinical suspicion of a papillary fibroblastoma or other primary cardiac tumors. At operation, after a median sternotomy, cardiopulmonary bypass via aortobicaval cannulation was established. An incision was made in the left atrium, and 


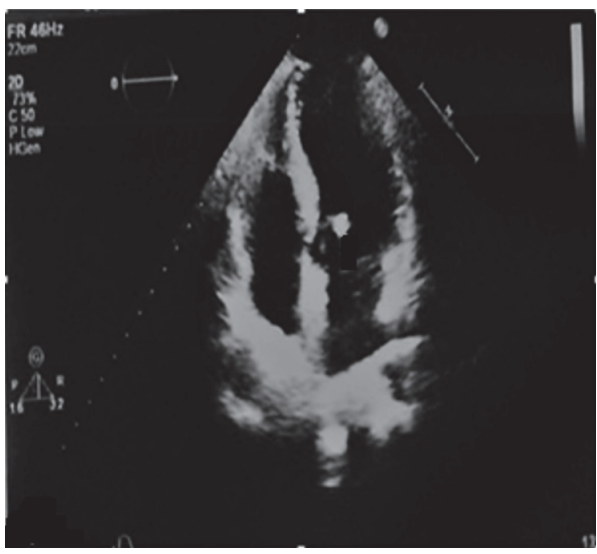

(a)

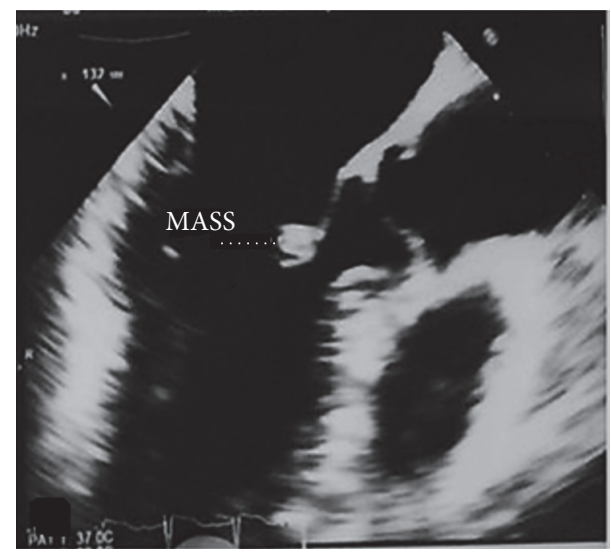

(b)

FIGURE 1: Transesophageal echocardiography showing an echogenic, round, and mobile mass, attached to the atrial surface of the anterior mitral valve leaflet by a short pedicle, measuring $5 \times 5 \mathrm{~mm}$.

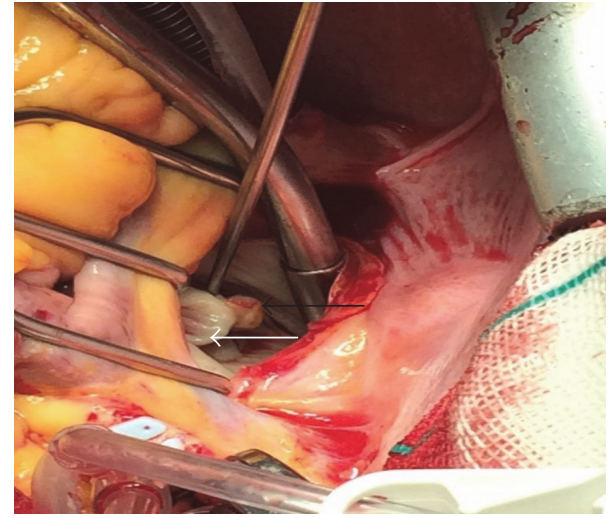

FIGURE 2: Intraoperative findings evidencing calcified tumor (black arrow) attached to the atrial surface of the anterior leaflet (white arrow) of the mitral valve.

a soft, firm, and friable mass was founded on the atrial surface of the anterior leaflet of the mitral valve which was easily removed (Figure 2). The anterior leaflet was repaired with an autologous pericardium patch. The patient was warmed and weaned from cardiopulmonary without difficulty.

Histopathological evaluation of the resected tumor showed a dense calcification (shredded due to no decalcification) in a background of amorphous degenerating fibrinous material (Figure 3) and according to the clinical and histological features, a diagnosis of cardiac CAT was provided. The postoperative course was uneventful, and the patient was discharged from the hospital 4 days after the surgery without any complications.

\section{Discussion}

Intracardiac masses have been classified as neoplastic and nonneoplastic lesions. Cardiac tumors are also divided into benign (about 75\%) and malignant neoplasms in which more than half of the benign tumors are myxomas [4]. Cardiac

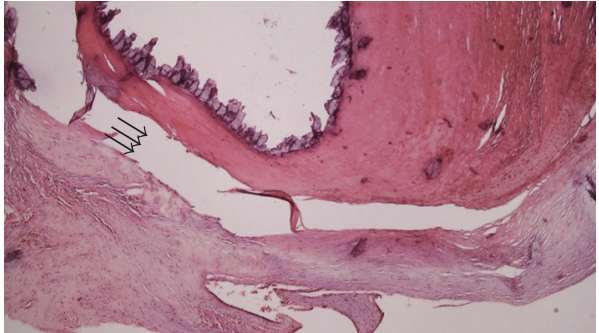

FIGURE 3: Histological examination showed dense calcification (shredded due to no decalcification) (arrows) in a background of amorphous degenerating fibrinous material (hematoxylin and eosin stain; $\times 40)$.

CAT which recently is described as a distinct pathological entity is extremely rare cardiac benign nonneoplasm [1]. The clinical manifestations of CAT are not specific and similar to those of other cardiac masses, often related to embolization or flow obstruction of the calcified fragments. Thus, differentiation between various types of cardiac masses, such as myxomas or fibroma, calcified thrombi, emboli, vegetation, and other intracardiac tumors may be difficult. The most common symptoms of these masses are progressive dyspnea, chest pain, syncope, and symptoms related to embolism [2, $3,5]$. Rarely, they may also present with cardiac arrhythmias such as ventricular tachycardia [3]. Further, mobile CATs have a higher chance of embolic event than immobile lesions. Even though modern cardiac imaging modalities can help to narrow down the differential diagnosis, surgical excision of the lesion and histological examination are necessary for definitive diagnosis [6]. Histological features of cardiac CATs consist of nodular calcium deposits within a background of fibrin or amorphous fibrillar material. Although they may occur in any chamber of the heart, they are found most commonly in the left ventricle (31.25\%) and mitral valve (25\%). Approximately $12.5 \%$ of cases of the cardiac CATs originated from the right atrium [7]. In our case, cardiac CATs were mobile and found in the mitral valve with initial presentation 
of TIA. The average age at diagnosis is 51 years with a range from 18 to 78 years. Prevalence is slightly more frequently in females [7]. The patient in our report was a 37-year-old woman. Although the exact pathogenesis of cardiac CAT is not determined, some authors believe the hypothesis that cardiac CAT is an organized and calcified mural thrombus [8]. Kawata et al. also suggested that disorders of calciumphosphorus metabolism in patients with renal dysfunction can be potential causes of CAT [9]. On the other hand, the lack of such predisposing conditions in other cases, like the current case, proposes that thrombosis may not be the only pathogenetic mechanism and also other various mechanisms can be involved. Surgical excision is mandatory because of the risk of complications or embolization and also for accurate diagnosis. Recurrence and fatal outcomes of cardiac CATs have rarely been reported after surgical resection, and, consequently, long-term follow-up with cardiac imaging studies, especially in patients with incomplete resection, is required $[6,10]$. In our case, the initial diagnosis was thought to be fibroblastoma or other primary cardiac tumors, but after resection of the tumor and histopathological evaluation, the diagnosis of CATs was confirmed. To summarize, we reported a case of CATs on the atrial surface of the anterior mitral valve leaflet in a 37-year-old female which was confirmed by histopathological examination after surgical removal.

\section{Conclusion}

The CATs of the heart are extremely rare nonneoplastic intracardiac mass and accurate diagnosis of a cardiac mass is often made on surgical excision and histological examination for the best management of them.

\section{Competing Interests}

The authors declare that they have no competing interests.

\section{References}

[1] C. Reynolds, H. D. Tazelaar, and W. D. Edwards, "Calcified amorphous tumor of the heart (cardiac CAT)," Human Pathology, vol. 28, no. 5, pp. 601-606, 1997.

[2] F. Sabzi, H. Karim, B. Eizadi, R. Faraji, and N. Javid, "Calcified amorphous tumor of the heart with purple digit," Journal of Cardiovascular and Thoracic Research, vol. 6, no. 4, pp. 261-264, 2014.

[3] N. Hussain, N. Rahman, and A. Rehman, "Calcified amorphous tumors (CATs) of the heart," Cardiovascular Pathology, vol. 23, no. 6, pp. 369-371, 2014.

[4] M. Esmaeilzadeh, "Echocardiographic evaluation of intracardiac masses," Journal of Tehran University Heart Center, vol. 3, no. 2, pp. 59-76, 2008.

[5] Y. Nazli, N. Colak, I. A. Atar et al., "Sudden unilateral vision loss: arising from calcified amorphous tumor of the left ventricle," Texas Heart Institute Journal, vol. 40, no. 4, pp. 453-458, 2013.

[6] M. E. Fealey, W. D. Edwards, C. A. Reynolds, P. A. Pellikka, and J. A. Dearani, "Recurrent cardiac calcific amorphous tumor: the CAT had a kitten," Cardiovascular Pathology, vol. 16, no. 2, pp. 115-118, 2007.
[7] E. K. Choi, J. Y. Ro, and A. G. Ayala, "Calcified amorphous tumor of the heart: case report and review of the literature," Methodist DeBakey Cardiovascular Journal, vol. 10, no. 1, pp. 3840, 2014.

[8] H. Seo, H. Fujii, T. Aoyama, and Y. Sasako, "Cardiac calcified amorphous tumor in a hemodialysis patient," Asian Cardiovascular and Thoracic Annals, vol. 24, no. 5, pp. 461-463, 2014.

[9] T. Kawata, H. Konishi, A. Amano, and H. Daida, "Wavering calcified amorphous tumour of the heart in a haemodialysis patient," Interactive Cardiovascular and Thoracic Surgery, vol. 16, no. 2, pp. 219-220, 2013.

[10] M. Lewin, S. Nazarian, J. E. Marine, D. D. Yuh, P. Argani, and M. K. Halushka, "Fatal outcome of a calcified amorphous tumor of the heart (cardiac CAT)," Cardiovascular Pathology, vol. 15, no. 5, pp. 299-302, 2006. 


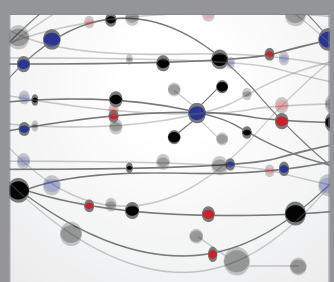

The Scientific World Journal
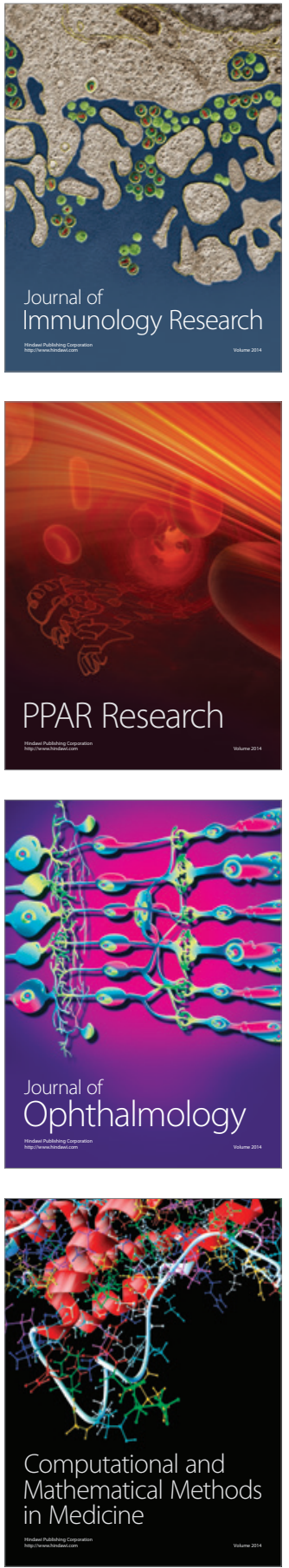

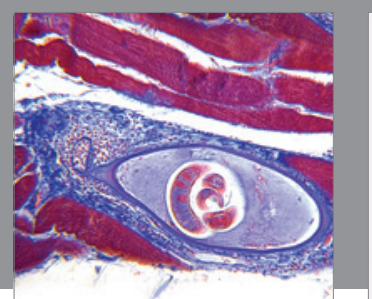

Gastroenterology Research and Practice
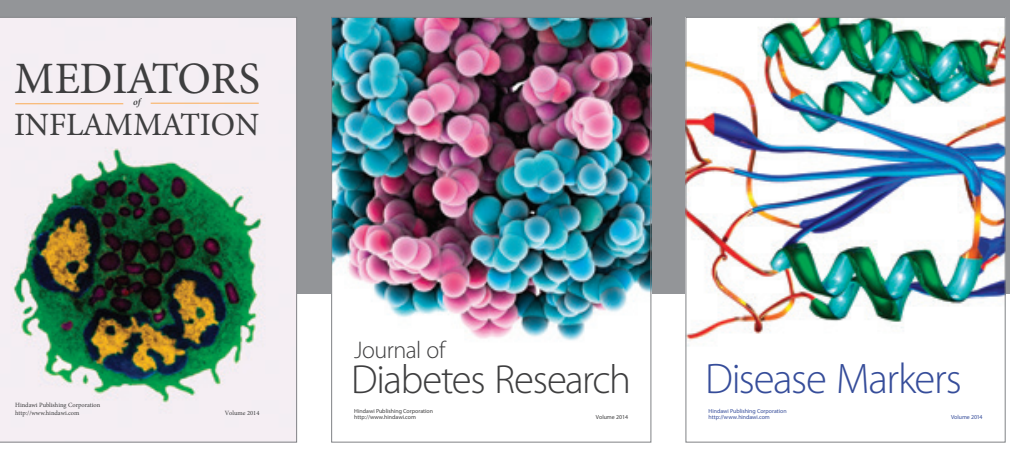

Disease Markers

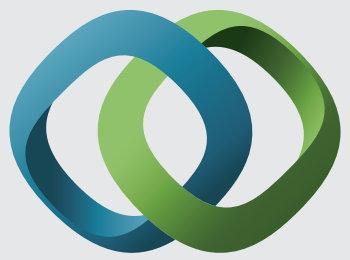

\section{Hindawi}

Submit your manuscripts at

https://www.hindawi.com
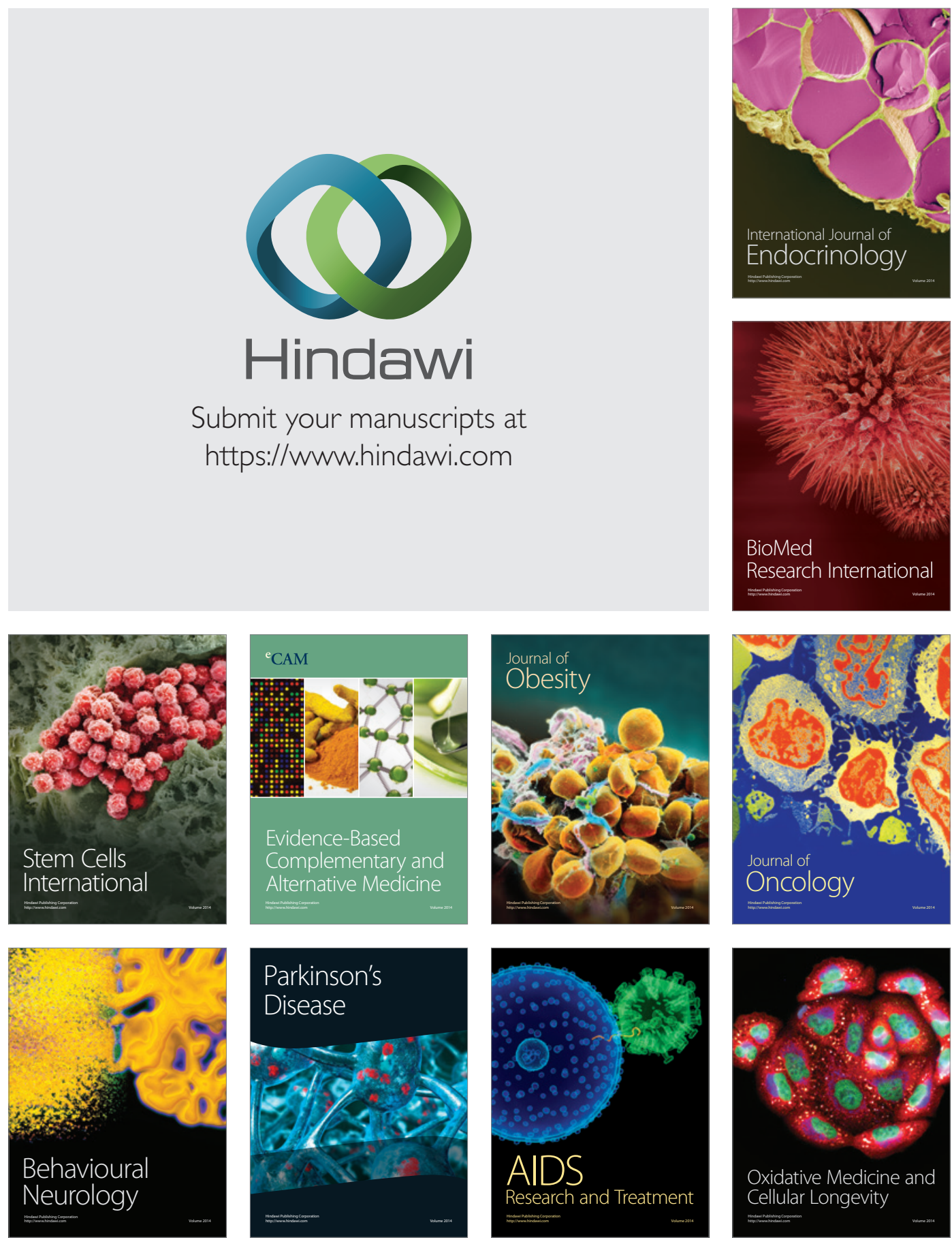